\title{
An explorative study of the ecological design of residence architecture in Ming and Qing dynasties in Yangzhou
}

\author{
Man Lu',2,a , Gang Wang ${ }^{3}$ and Jian Zhou ${ }^{1}$ \\ ${ }^{1}$ School of Civil and Transportation, Hohai University, Nanjing, Jiangsu Province, 210098, China \\ ${ }^{2}$ School of Architecture, Southeast University, Nanjing, Jiangsu Province, 210096, China \\ ${ }^{3}$ Nanjing Gulou Audit office, Nanjing, Jiangsu Province, 210008, China
}

\begin{abstract}
Presently ecologicalization has become a key aspect of architecture designs. As studies deepen, the bountiful ecological ideas and design methods represented in Chinese traditional architectural culture are increasingly valued. There remain as of today quite a few residence mansions built in Ming and Qing dynasties in Yangzhou- a city famous for its history and culture. The distinctive regional culture and architectural style that those architectures embody has received worldwide attention in the academic field. By weighing on the shaping of architectural forms, choice in connection genres and structuring measures as well as sorting through and studying on the ecologicalized architectural design practices such as reemploying local materials, deeper understanding of traditional architecture will be achieved for us to better inherit and develop traditional architectural culture. Hence in-depth studies on contemporary ecological architecture and sustainable development will be of greater practical significance. Furthermore, it is beneficial to expand ideas on ecological design, truly integrate architectures into the setting to give expression to the ecological and culture features of them and provide theoretic support for creating new architecture with diversified culture implications and ecological features in light of actual conditions in an orderly manner.
\end{abstract}

\section{Ecologization and its practical significance in traditional Chinese architecture}

So called ecologic architecture is theories and methodologies on architectural design and planning based on ecology that studies the symbiotic relationship between creatures in nature and the environment; in other words, it is a branch of ecology that explores the balanced development of life activities and functions on earth into architecture, reflecting the value orientation of modern architecture community [1]. Chinese ancient philosophy has early on realized that humans and nature are an inseparable whole, emphasized that the relationship between humans and nature is defined by the connections between them. The Chinese philosophic concept of "nature and humans being oneness" is thus a view of plain ecology. Chinese ancient philosopher Lao zi said "Humans follows earth, earth follows heaven, heaven follows Dao, Dao follows naturalness". The saying indicates that humans and nature all have their own order and ultimately follow the ever natural laws. While nature

\footnotetext{
${ }^{\mathrm{a}}$ Corresponding author : mmmm0526@sina.com
} 
is all inclusive and acts as the object, humans coexist with nature and acts as the subject. "Nature and humans being oneness" asserts that the subject integrates with the object into a whole. Nature is seen as the greater entity and the human as a smaller entity. Human life continues as it is in the constant exchanges of energy and information with nature. Heaven, earth and humans are an integrated ecologic system featuring organic circulation and metabolism. The ecologic view represented in traditional Chinese architecture is a reflection of traditional Chinese views, aesthetic concept and technology on architecture.

The traditional Chinese residence architectures are the emnodiment of ecological residence. Residents of all ethnic groups in all areas have taken factors into full consideration such as local natural environment, climate, geology, land form, architecture materials, technology, customs and living habits. Ancient Chinese architecture plays a positive role in the harmony of ancient ecosystem, from which we could find many traces of primeval ecological architectures including: the organic close combination between ancient Chinese architectures and the surroundings; the courtyard layout of architectures is consistent with family structure (the consistency on the level of materials and spirit); the architecture structure is sustainable with individual components fixable and replaceable when broken; most of materials for constructing houses are local, which saves energy and reduces pollution. All of the above are of great practical significance for and shed light on the current ecologic architectural technique studies.

\section{Ecologic design strategy in the planning of residential architecture of Yangzhou in Ming and Qing dynasty}

\subsection{Location selection and layout}

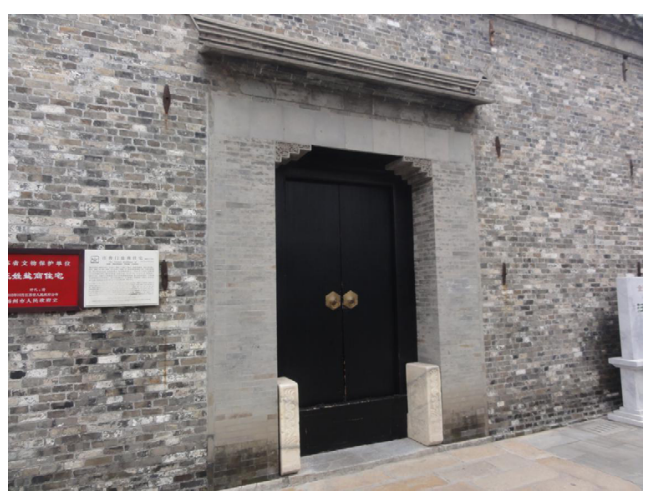

Figure 1. Outer facade of Lumen Wang Mansion.

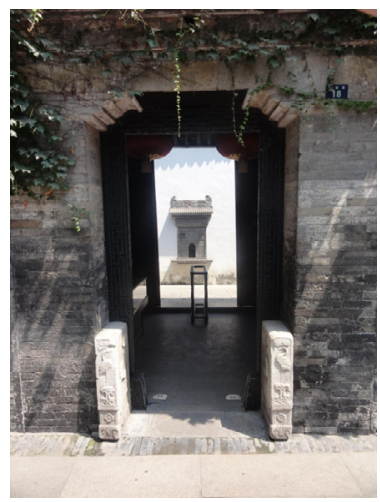

Figure 2. The entrance gate and shrine of Wangshi Garden.

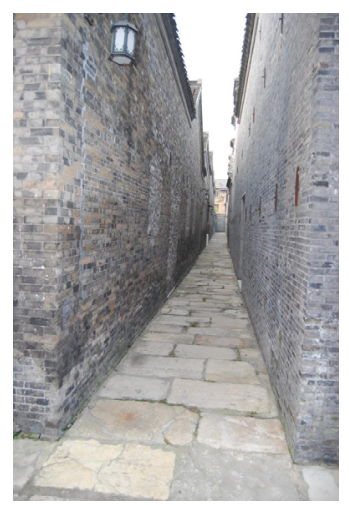

Figure 3. Fire preventing alley.

Houses built in Ming and Qing dynasties in Yangzhou are found in both the old and new towns just as Qinglong- one emperor in Qing dynasty described "most salt merchants live in the new town while the old habitants live in the old town" [2]. However, these mansions were often chosen to be built close to the marketplace or along the river to save commute cost. The residential architecture built in the busy marketplace use high walls to block the noise from the outside and protect the privacy by installing no window on the outer wall (Fig 1). Jicheng (a famous landscape architect in Ming Dynasty) wrote in his book Yuanye "though living in the neighborhood of bustle, no noise can be heard behind the closed door" [3]. Furthermore, the entrance gate is not on the same longitudinal axis with the etiquette gate with the former usually located towards the southeast corner of the mansion. Upon entering the mansion, one will be faced with a solid wall with a shrine embedded in it (Fig 2). The sound from the streets are blocked or reflected by the wall, which ensures the quietness within the house. As to the 
layout, the front and middle parts of the mansion are the space for meeting guests while the rear and bilateral parts are for living functions. With the locations of the mansions soundly planned according to the local situation, the mansions coexist with each other without losing their independence. Under the condition of not exceeding land quota, all residence architectures are built in parallel with firepreventing alleys built which not only helps prevent fire but also contributes to ventilation and taking the heat in summer. (Fig 3)

\subsection{Facing direction and spacing}

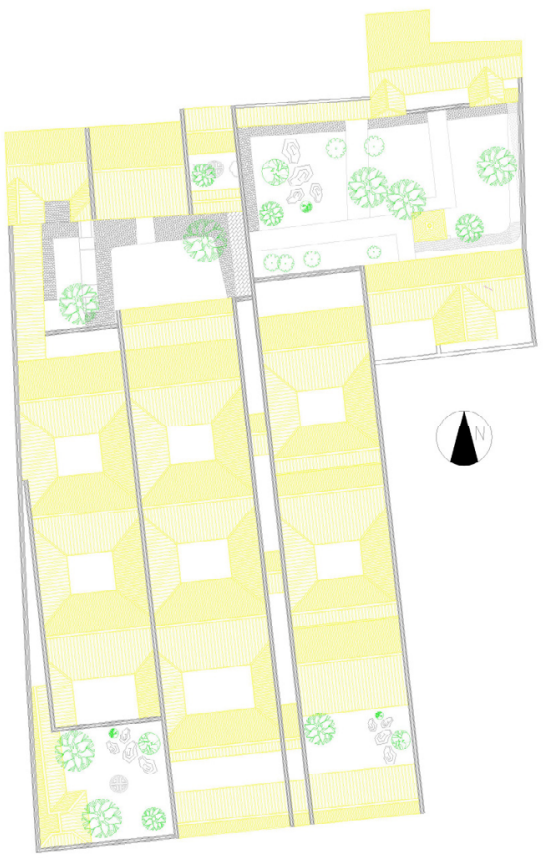

Figure 4. General layout of Wangshi Garden.

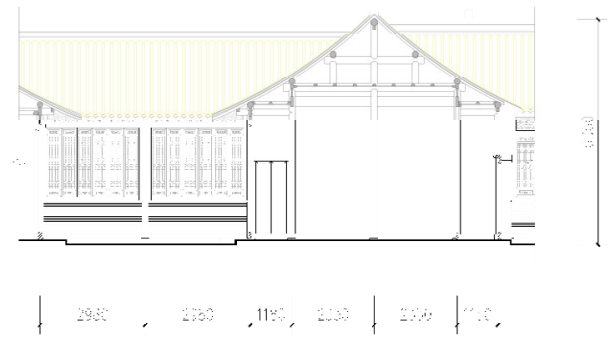

Figure 6. Fire preventing alley.

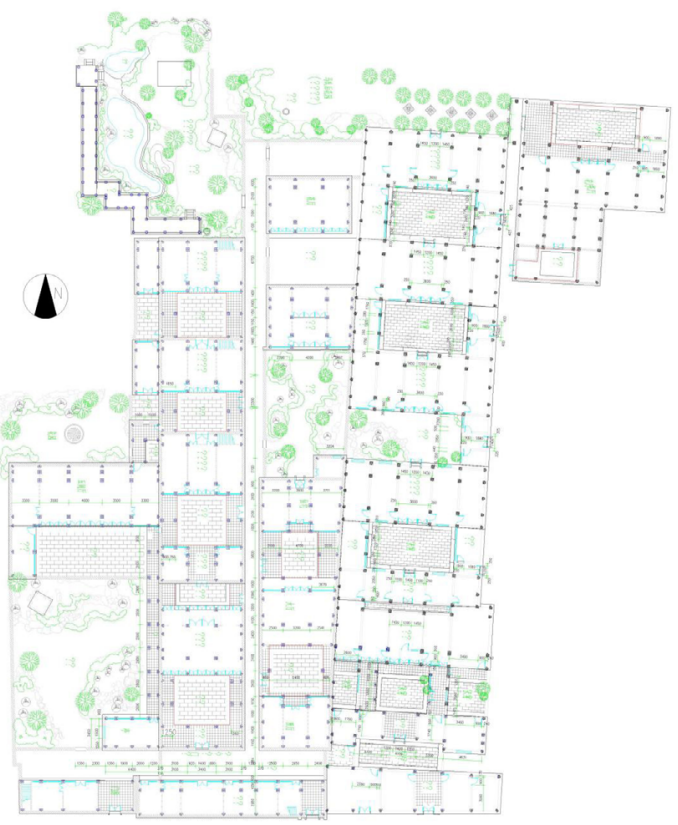

Figure 5. General layout of Dingshi and Mashi's Former Residence.

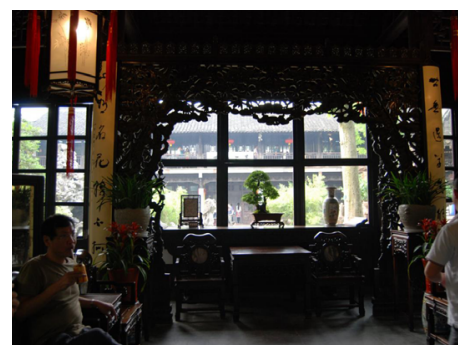

Figure 7. Yiyu Hall.

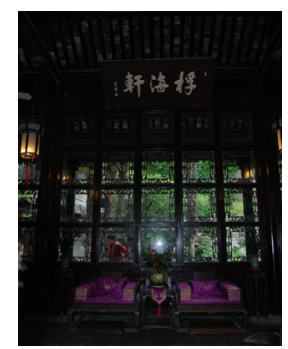

Figure 8. Fuhai Hall.

The ideal facing direction of architecture in Yangzhou area is between SE $15^{\circ}$ and SW $15^{\circ}$. Research shows that houses built in Ming and Qing Dynasty all met such a standard, typically represented by Ge Garden, Wangshi Garden (Fig 4), Dingshi and Mashi's Former Residence (Fig 5). Even for those mansions that are subject to restrictions of land size, the facing direction of gate or the layout of the house is adjusted to ensure that the architecture has a sound facing direction.

Although there were many Huizhou businessmen living there in Yangzhou in Ming and Qing dynasty and Huizhou style architecture are common in the residential architectures in Yangzhou, quite 
a few differences are found to exist between them in the field research. For example, the length of houses in Yangzhou area is at a ratio of 1:1 to the height of the house (Fig 6). The width of the architect is larger than its length, which makes the architect a flat rectangle to ensure ample sunlight and ventilation. On the other hand, Huizhou style residence features narrow spacing between courtyards while Yangzhou residences are more spacious.

\section{Ecological design strategies in construction of residential architectures of Yangzhou in Ming and Qing dynasty}

\subsection{Carpentry system in frame structure}

Carpentry system in the residential architectures of Yangzhou in Ming and Qing dynasty can be divided into three types: post- and- lintel, cross-and-tie and the combination of both which is most common. Post-and-lintel construction can provide broader indoor space but costs more wood; crossand-tie construction features sound integrity and economic wood usages but requires dense arrangement of pillars; the combination combines the advantages of both, which makes it ideal for constructing sound, cost effective and spacious indoor space.

Carpentry system gets rid of walls and enhances the connections between the architect and environment. For example, four-sided halls for scenery viewing purpose such as Yiyu Hall of Ge Garden (Fig 7) and Fuhai Hall of He Garden (Fig 8) have windows on four sides. When sitting inside the halls, one is overwhelmed by the views of four seasons from four directions. It also makes it possible to partition the indoor space more freely. For example, a single space can be divided into four or five when it appears to have only three; or use varied kinds of screen panels to create personalized space.

Furthermore, wood structure is easy to maintain. Despite its short longevity caused by years of rotting, mortise and tenon joint are easy to uninstall thus making maintenance easier; also due to certain level of mobility of mortise and tenon joint, wood structure might tilt under foreign force but not fall easily, therefore it has strong anti damage ability. Almost all the components in the carpentry system can be replaced individually, which reduces maintenance cost significantly and extends the longevity of the architecture.

\subsection{Walls and flooring featuring thermal insinuation}

"He He Wall" of Yangzhou has distinct local characteristics. Referred to as "clear water good" by the locals, the walls, as thick as $360 \mathrm{~mm}$, are constructed with bricks and mortar without whitewash, which requires advanced craftsmanship. Such walls are better at thermal insulation than average brick walls. The lower section of the wall is built with flat laid bricks and mortar while the above section built with rowlock laid bricks. This way of laying bricks symbolizes "harmony" in the cultural sense and saves materials. When building such a wall, crosspiece and "mu pai shan" (a wooden consolidating structure)placed within the wall, along with "tie ba ju"(an iron consolidating structure) shaped like the Chinese character "丁" and nailed on the outside, together consolidate the wall body, equivalent to ring beam consolidating in modern concrete construction[4].

Due to the humid climate in summer in Yangzhou area, the indoor floorings would easily get moist, thus residential houses built in Ming and Qing dynasties in Yangzhou all adopted moist preventive measures. Usually the halls use quadrel flooring with metal bowl at the bottom layer. The four corners of the quadrel are placed on the inverted bowel, which smoothes the edges of the quadrel and prevents the moist. The bedrooms have better absorbing wooden floorings with metal bowls also placed underneath in addition to vent holes installed along the outer wall, which helps expel the moist and slows the process of rotting away wood floor. In some cases, wooden floorings are added to the quadrel layer so as to make the room warmer in winter and removed in summer to make it cooler. 


\subsection{Flexible arrangement of door and windows}

As frame structures in carpentry system needs no wall to bear the weight, the arrangement of windows can be quite flexible. Generally the middle room in the south of the house has 6 or 8 lattice doors [5] and the room next to it often uses lattice windows. As they cover large area, it helps to enhances the lighting indoors and capture warmth from sunlight in winter. In summer, the wooden barrier under the lattice window could be removed leaving only hollowed paneled railings behind. Together with lattice door, screen panel and wicket, they contribute to natural cross ventilation thus cool down the indoors. The northern part of the house has no windows installed for privacy considerations. For architectures mainly for scenery viewing purpose, they often have openings on the north and south sides while the four sided hall even has windows on all four sides with the French window installed on the south side, which ensures sound indoor lighting and ventilation. The way of constructing houses in Ming and Qing dynasty in Yangzhou did not remain the same as time passed. As an immigration city, the inclusiveness of Yangzhou is also represented in its architecture. For example, the blind window and blind door (Fig 9) in Yuxiu Tower of He Garden boldly adopted western elements which not only better adjusts temperature and lighting indoors (Fig 11) but also protects the privacy of family members.

\section{Ecological design strategies in construction of residential architectures of Yangzhou in Ming and Qing dynasty}

\subsection{Arrangement of local plants}

After long time of evolution, local plants, a representation of local features, have gained strong life force and are the fittest for local natural conditions. The local plants grown in the houses of Yangzhou in Ming and Qing dynasties include: Weeping willow, ginkgo, maple, magnolia, Koelreuteria, hackberry, Sophora japonica, elm, privet, Viburnum, osmanthus, loquat, crape myrtle, wintersweet, Chinese rose, peony, rochebruni, water lilies and varied kinds of bamboo, most of which are in sound growth. The strategy to grow local plants is not only economic and beneficial for their survival, but also the significant one in the ecological design of contemporary architectural landscape.

The vegetation system composed of trees, shrubs and grass constitutes a multi-layer ecologic community with the help of well-arranged architecture, waters, rockery and paths. The examples of vertical greening strongly promoted in contemporary ecological designs are present in houses of Yangzhou built in Ming and Qing dynasties. For example, the book loft of Ge Garden takes advantage of the climbing and adhesive properties of Parthenocissus tricuspidata by using it to ornament the outer wall with its dense green leaves. In summer, the flourishing leaves of Parthenocissus tricuspidata can shade the wall to cool down indoors (Fig 10 Book loft of Ge Garden). In winter, the concentrated branches and twigs of Parthenocissus tricuspidata after leaves falling could also form an insinuating layer to help the architecture keep the warmth inside.

\subsection{Flexible drainage system as situation dictates}

Traditional architecture in Yangzhou relies on natural drainage system. As rain fall from the roof to the ground paved with slates, bricks and pebbles, it seeps into the soil layer on its own, only contributing to the replenishment of underground water but also reducing the heat island effect and adjusts microclimate.

The surface of passage space such as fire preventive alleys is paved with bricks whose direction of arrangement is unparallel to walking direction, which somewhat prevent people from slipping. Moreover as the middle section of the path is higher than either part, it is conductive to rapid discharge of rainwater. For thunderstorms with large precipitation, natural seeping is not quick enough to discharge rainwater falling on the surface of the yard. In this situation, outfalls can be installed (Fig 
11 Outfall). All those mentioned above can be applied to the construction of modern ecological residential area.

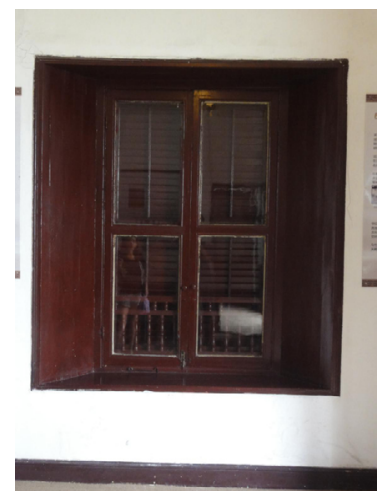

Figure 9. The blind window and blind door

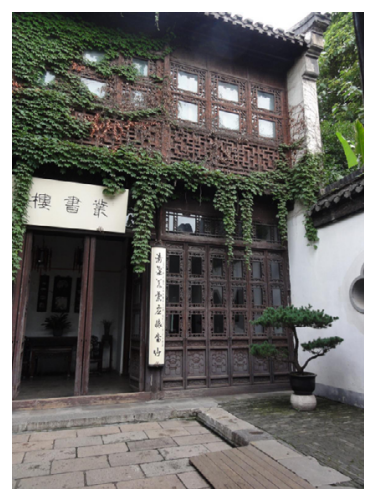

Figure 10. Book loft of Ge Garden

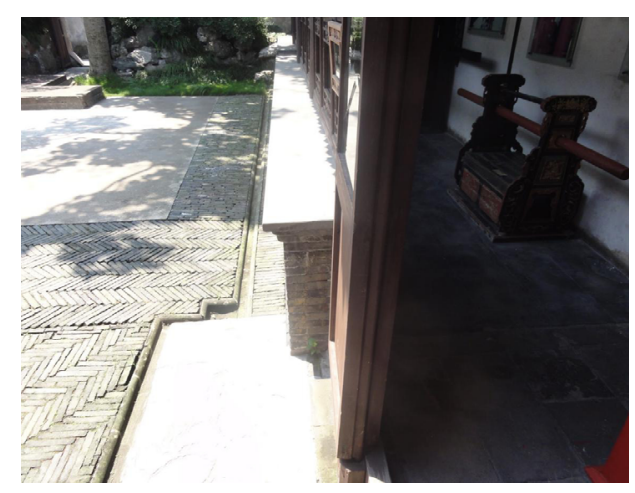

Figure 11. Outfall

\section{Conclusion}

Residential architecture built in Ming and Qing Dynasties is a vital part of traditional culture of Yangzhou. The unique style grants it the important status in Chinese regional architecture. Under the guidance of philosophical view "nature and human being oneness", Yangzhou residence architecture creates refined living environment compliant with local climate, economic and technological conditions by learning from nature and smart arrangement of architecture elements. In terms of planning, construction and landscape design, it reasonably uses the resources available to create sound micro ecologic community.

In the modern residential environmental design, the method of separating the static and dynamic sections should be widely used, leading a series of space experience — - from pleasant shared space previously to groups before reaching the living area.Green shared space in residential areas can not only regulate the micro-climate, but also get dwellers relieved physically. Additionally, it creates a positive space for self-monitoring of the neighborhood resulting in ecological settlements.Modern constructing techniques and materials make it possible to prefabricate houses, whose structural system is parallel to the one of Yangzhou traditional architecture. In fact, the life-span will be extended as each component can be selected or replaced. Moreover, the cost of enclosure will be reduced leading to residential ecologicalization.It is an inevitable trend for ecological building to choose refined local materials, which can save the expenditure of transportation and maintenance and reflect the characteristics in context to arouse a sense of belonging. Such ecology is both in physical universe and on psychological level.

To salute and analysis the traditional architecture, the essence of ecological and livable concepts ought to be selected and merge with advanced technology to create a suitable living space for modern life, rather than adding symbolic elements to new buildings awkwardly. The summary of the experiences in all the above respects provides reference for contemporary ecological design and also helps promote residential architectural culture of Yangzhou.

\section{References}

1. B. Edwards, Sustainable Architecture (Trans v), 3-324 (Chinese architecture publishing house, Beijing, 2004)

2. C.H. Zhang, Studies on the Development Path of Residence Architecture in Yangzhou Area, $\mathbf{5 0}$ (Southeast University Publishing House, 2011) 
3. J. Cheng and Z. Chen, Garden Construction, 60 (Chinese architecture Industry Publishing House, Beijing, 1988)

4. J. Yang and H. Du, The Charm of Ge Garden, 59 (Yangzhou Guangling Publishing House, 2006)

5. Lattice Door: The use of lattice door dates back to the late Tang Dynasty. Its lower part is a solid water-proof board (apron) while the upper one is hollow for forming a light transmission part. And it replaced board door for its nice lighting performance. 